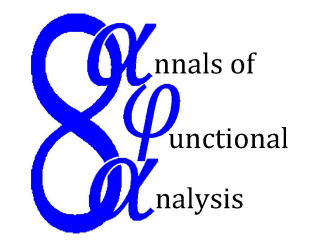

Ann. Funct. Anal. 5 (2014), no. 1, 101-108

$\mathscr{A}$ NNALS OF $\mathscr{F}$ UNCTIONAL $\mathscr{A}$ NALYSIS

ISSN: 2008-8752 (electronic)

URL:www.emis.de/journals/AFA/

\title{
MAJORIZATION OF SINGULAR INTEGRAL OPERATORS WITH CAUCHY KERNEL ON $L^{2}$
}

\author{
TAKANORI YAMAMOTO
}

This paper is dedicated to Professor Tsuyoshi Ando

Communicated by C. P. Niculescu

\begin{abstract}
Let $a, b, c$ and $d$ be functions in $L^{2}=L^{2}(\mathbb{T}, d \theta / 2 \pi)$, where $\mathbb{T}$ denotes the unit circle. Let $\mathcal{P}$ denote the set of all trigonometric polynomials. Suppose the singular integral operators $A$ and $B$ are defined by $A=a P+b Q$ and $B=c P+d Q$ on $\mathcal{P}$, where $P$ is an analytic projection and $Q=I-P$ is a co-analytic projection. In this paper, we use the Helson-Szegö type set $(H S)(r)$ to establish the condition of $a, b, c$ and $d$ satisfying $\|A f\|_{2} \geq\|B f\|_{2}$ for all $f$ in $\mathcal{P}$. If $a, b, c$ and $d$ are bounded measurable functions, then $A$ and $B$ are bounded operators, and this is equivalent to that $B$ is majorized by $A$ on $L^{2}$, i.e., $A^{*} A \geq B^{*} B$ on $L^{2}$. Applications are then presented for the majorization of singular integral operators on weighted $L^{2}$ spaces, and for the normal singular integral operators $a P+b Q$ on $L^{2}$ when $a-b$ is a complex constant.
\end{abstract}

\section{INTRODUCTION}

Let $m$ denote the normalized Lebesgue measure $d \theta / 2 \pi$ on the unit circle $\mathbb{T}=$ $\{|z|=1\}$. For $0<p \leq \infty, L^{p}=L^{p}(\mathbb{T}, m)$ denotes the usual Lebesgue space on $\mathbb{T}$ and $H^{p}$ denotes the usual Hardy space on $\mathbb{T}$. Let $S$ be the singular integral operator defined by

$$
(S f)(\zeta)=\frac{1}{\pi i} \int_{\mathbb{T}} \frac{f(\eta)}{\eta-\zeta} d \eta \quad(\text { a.e. } \zeta \in \mathbb{T})
$$

Date: Received: 1 July 2013; Accepted: 16 September 2013.

2010 Mathematics Subject Classification. Primary 45E10; Secondary 47B35.

Key words and phrases. Analytic projection, singular integral operator, majorization, weighted norm inequality, $A_{2}$-weight, Helson-Szegő weight, Hardy space. 
where the integral is understood in the sense of Cauchy's principal value (cf. [6], Vol. 1, p.12). If $f$ is in $L^{1}$ then $(S f)(\zeta)$ exists for almost all $\zeta$ on $\mathbb{T}$. Let

$$
P=(I+S) / 2 \text { and } Q=(I-S) / 2,
$$

where $I$ denotes the identity operator. Then $P z^{n}=0$ if $n<0$, and $P z^{n}=z^{n}$ if $n \geq 0 . \quad P$ is said to be an analytic projection or the Riesz projection. Let $\mathcal{P}_{1}=\operatorname{span}\left\{z^{n}: n \geq 0\right\}$ be the set of analytic polynomials, and let $\mathcal{P}_{2}=\overline{z \mathcal{P}_{1}}=$ $\operatorname{span}\left\{z^{n}: n<0\right\}$. Then $Q=I-P, P\left(f_{1}+f_{2}\right)=f_{1}$ and $Q\left(f_{1}+f_{2}\right)=f_{2}$ for all $f_{1} \in \mathcal{P}_{1}$ and $f_{2} \in \mathcal{P}_{2} . Q$ is said to be a co-analytic projection. Let $\alpha, \beta \in L^{\infty}$, and $W$ is a nonnegative function in $L^{1}$. In [8] and [16], the condition of $\alpha, \beta$ and $W$ such that $\alpha P+\beta Q$ is contractive was given. In [9], the conditions of $\alpha, \beta$ and $W$ such that $\alpha P+\beta Q$ is bounded and bounded below was given. In [10] and [11], for $\alpha, \beta \in L^{\infty}$, the norm formula of $\alpha P+\beta Q$ on the weighted $L^{2}$ space was given. In [10], [11] and [16], the another proofs of Feldman-KrupnikMarkus's theorem ([6], Vol. 2, p.213, Theorem 5.1, and p.215, Lemma 5.3) were given. In this paper, for $a, b, c, d \in L^{2}$, we consider the singular integral operators $A=a P+b Q$ and $B=c P+d Q$. If $a, b, c, d \notin L^{\infty}$, then $A$ and $B$ are unbounded. In Section 2, we use the Helson-Szegö type set $(H S)(r)$ to establish the condition of $a, b, c$ and $d$ satisfying $\|A f\|_{2} \geq\|B f\|_{2}$ for all $f$ in $\mathcal{P}$. The main theorem is Theorem 2.4. If $a, b, c, d \in L^{\infty}$, then $A$ and $B$ are bounded, and this is equivalent to that $B$ is majorized by $A$ on $L^{2}$, i.e., $A^{*} A \geq B^{*} B$ on $L^{2}$. As an application of Theorem 2.4, we have Theorem 2.5. In Section 3, some applications are presented for the majorization of singular integral operators on weighted $L^{2}$ spaces, and for the normal singular integral operators $a P+b Q$ on $L^{2}$ when $a-b$ is a complex constant.

\section{MAIN THEOREM}

In this section, we use the Helson-Szegö type set $(H S)(r)$ to establish the condition of $a, b, c$ and $d$ satisfying $\|A f\|_{2} \geq\|B f\|_{2}$ for all $f$ in $\mathcal{P}$. The main theorem is Theorem 2.4. If $a, b, c, d \in L^{\infty}$, then this is equivalent to that $B$ is majorized by $A$ on $L^{2}$, i.e., $A^{*} A \geq B^{*} B$ on $L^{2}$. By Douglas's criterion (cf. [4], [14], p.2), this implies that there is a contraction $C$ on $L^{2}$ such that $B$ is factorized as $B=C A$. Let $\tilde{f}$ denote the harmonic conjugate function of $f \in L^{1}$. Then $S f=i \tilde{f}+\int_{\mathbb{T}} f d m$. It is well known that the Helson-Szegö set

$$
(H S)=\left\{e^{u+\tilde{v}} ; u, v \in L^{\infty} \text { are real functions, and }\|v\|_{\infty}<\frac{\pi}{2}\right\}
$$

is equal to the set of all Muckenhoupt $\left(A_{2}\right)$-weights (cf. [5], p.254).

Definition 2.1. For a function $r$ satisfying $0 \leq r \leq 1$ and $\int_{\mathbb{T}} r d m>0$, we define the Helson-Szegö type set $(H S)(r)$ :

$$
\begin{gathered}
(H S)(r)=\left\{\gamma e^{u+\tilde{v}} ; \gamma \text { is a positive constant, } u, v\right. \text { are real functions, } \\
\left.u \in L^{1}, v \in L^{\infty},|v| \leq \pi / 2, r^{2} e^{u}+e^{-u} \leq 2 \cos v\right\}
\end{gathered}
$$

If $|v| \leq \pi / 2$, then $e^{\tilde{v}} \cos v \in L^{1}$ (cf. [5], p.161), and hence $e^{-\tilde{v}} \cos v \in L^{1}$. Therefore $(H S)(r) \subset\left\{W: W>0, r^{2} W \in L^{1}, W^{-1} \in L^{1}\right\}$. If $r^{-1} \in L^{\infty}$, then $(H S)(r) \subset(H S)$. In [9], we defined the another Helson-Szegö type set which is 
similar to $H S(r)$. We use $H S(r)$ to study the majorization of singular integral operators.

Lemma 2.2. Let $W$ be a non-negative function in $L^{1}$, and let $\phi$ be a function in $L^{1}$. Suppose $|\phi| \geq W$ and $\int_{\mathbb{T}}(|\phi|-W) d m>0$. Then the following conditions (1) (3) are equivalent.

(1) There is a $k$ in $H^{1}$ such that $|\phi-k| \leq W$.

(2) There is a non-zero $k$ in $H^{1}$ such that $|\phi-k| \leq W$.

(3) $\log |\phi| \in L^{1}$ and there is a $V$ in $(H S)(r)$ such that $\phi / V$ is in $H^{1 / 2}$, where $r=|\phi|^{-1} \sqrt{|\phi|^{2}-W^{2}}$.

Proof. (1) $\Rightarrow(2)$ : By (1), if $k=0$, then $0 \leq|\phi|-W \leq 0$, and hence $|\phi|=W$. This is a contradiction. Therefore $k \neq 0$.

$(2) \Rightarrow(3): \operatorname{By}(2),|\phi-k| \leq W \leq|\phi|$, and hence $0<|k| \leq 2|\phi|$. Since $\log |k| \in L^{1}, \log |\phi| \in L^{1}$. Since

$$
\left|1-\frac{k}{\phi}\right|^{2} \leq \frac{W^{2}}{|\phi|^{2}}=1-\frac{|\phi|^{2}-W^{2}}{|\phi|^{2}}=1-r^{2} \leq 1,
$$

it follows that $\operatorname{Re}(k / \phi) \geq 0$. Since $\log |k / \phi| \in L^{1}$, it follows that there are real functions $u \in L^{1}$ and $v \in L^{\infty},|v| \leq \pi / 2$ such that $k / \phi=e^{-u-i v}$. Then $0 \leq r \leq 1$ and $\left|1-e^{-u-i v}\right|^{2} \leq 1-r^{2}$. Hence $r^{2}+e^{-2 u} \leq 2 e^{-u} \cos v$, and so $r^{2} e^{u}+e^{-u} \leq 2 \cos v$. Since $\phi=k e^{u+i v}$, it follows that $\phi e^{-u-\tilde{v}}=k e^{i v-\tilde{v}} \in H^{p}$, for some $p>0$. Since $e^{-u} \leq 2 \cos v$, it follows that $e^{-u-\tilde{v}} \leq 2 e^{-\tilde{v}} \cos v$. Since $|v| \leq \pi / 2, e^{-\tilde{v}} \cos v \in L^{1}$ (cf. [5], p.161). Hence $e^{-u-\tilde{v}} \in L^{1}$. Since $\phi \in L^{1}$, it follows that $\phi e^{-u-\tilde{v}} \in H^{1 / 2}$. $(3) \Rightarrow(1)$ : By (3), if $k=\phi e^{-u-i v}$, then $k=\left(\phi e^{-u-\tilde{v}}\right) e^{\tilde{v}-i v} \in H^{p}$, for some $p>0$. Since $|\phi-k|^{2}=|\phi|^{2}\left|1-e^{-u-i v}\right|^{2}=|\phi|^{2} e^{-u}\left(e^{u}+e^{-u}-2 \cos v\right)$ and $e^{u}+e^{-u}-2 \cos v \leq\left(1-r^{2}\right) e^{u}=|W / \phi|^{2} e^{u}$, it follows that $|\phi-k| \leq W$. Hence $|k| \leq|\phi|+W$, and so $k \in H^{1}$. This completes the proof.

Lemma 2.3. Let $W_{1}, W_{2}$ be real functions in $L^{1}$, and let $\phi$ be a function in $L^{1}$. Suppose $|\phi|^{2}-W_{1} W_{2} \geq 0$. Then the following conditions (1) and (2) are equivalent.

(1) For all $f_{1} \in \mathcal{P}_{1}$ and $f_{2} \in \mathcal{P}_{2}$,

$$
\left|\int_{\mathbb{T}} f_{1} \overline{f_{2}} \phi d m\right| \leq \frac{1}{2} \int_{\mathbb{T}}\left(\left|f_{1}\right|^{2} W_{1}+\left|f_{2}\right|^{2} W_{2}\right) d m
$$

(2) $W_{1} \geq 0, W_{2} \geq 0$, and either (a) or (b) holds.

(a) $|\phi|^{2}-W_{1} W_{2}=0$.

(b) $\log |\phi| \in L^{1}$, and there is a $V$ in $(H S)(r)$ such that $\phi / V$ is in $H^{1 / 2}$, where $r=|\phi|^{-1} \sqrt{|\phi|^{2}-W_{1} W_{2}}$.

Proof. (1) $\Rightarrow(2)$ : By Cotlar-Sadosky's lifting theorem [3], $W_{1} \geq 0, W_{2} \geq 0$, and there is a $k$ in $H^{1}$ such that $|\phi-k|^{2} \leq W_{1} W_{2}$. By Lemma 2.2, this implies (2).

$(2) \Rightarrow(1)$ : Suppose (a) holds. Then

$$
\begin{aligned}
\left|\int_{\mathbb{T}} f_{1} \overline{f_{2}} \phi d m\right| & \leq \int_{\mathbb{T}}\left|f_{1} f_{2} \phi\right| d m=\int_{\mathbb{T}}\left|f_{1} f_{2}\right| \sqrt{W_{1} W_{2}} d m \\
& \leq \frac{1}{2} \int_{\mathbb{T}}\left(\left|f_{1}\right|^{2} W_{1}+\left|f_{2}\right|^{2} W_{2}\right) d m
\end{aligned}
$$


This implies (1). Suppose $\int_{\mathbb{T}}\left(|\phi|^{2}-W_{1} W_{2}\right) d m>0$ and (b) holds. Then it follows from Lemma 2.2 that there is a $k$ in $H^{1}$ such that $|\phi-k|^{2} \leq W_{1} W_{2}$. Hence for all $f_{1} \in \mathcal{P}_{1}$ and $f_{2} \in \mathcal{P}_{2}$,

$$
\begin{aligned}
\left|\int_{\mathbb{T}} f_{1} \overline{f_{2}} \phi d m\right| & =\left|\int_{\mathbb{T}} f_{1} \overline{f_{2}}(\phi-k) d m\right| \\
& \leq \int_{\mathbb{T}}\left|f_{1} f_{2}\right| \cdot|\phi-k| d m \\
& \leq \int_{\mathbb{T}}\left|f_{1} f_{2}\right| \sqrt{W_{1} W_{2}} d m \\
& \leq \frac{1}{2} \int_{\mathbb{T}}\left(\left|f_{1}\right|^{2} W_{1}+\left|f_{2}\right|^{2} W_{2}\right) d m
\end{aligned}
$$

This implies (1). This completes the proof.

Remark A. In Lemma 2.3, if $|\phi|^{2}-W_{1} W_{2} \leq 0$ then

$$
\begin{aligned}
\left|\int_{\mathbb{T}} f_{1} \overline{f_{2}} \phi d m\right| & \leq \int_{\mathbb{T}}\left|f_{1} f_{2} \phi\right| d m \leq \int_{\mathbb{T}}\left|f_{1} f_{2}\right| \sqrt{W_{1} W_{2}} d m \\
& \leq \frac{1}{2} \int_{\mathbb{T}}\left(\left|f_{1}\right|^{2} W_{1}+\left|f_{2}\right|^{2} W_{2}\right) d m
\end{aligned}
$$

and so (1) holds without the condition (2).

Theorem 2.4. Let $a, b, c, d$ be functions in $L^{2}$. Then the following conditions (1) and (2) are equivalent.

(1) For all $f$ in $\mathcal{P}$,

$$
\int_{\mathbb{T}}|(a P+b Q) f|^{2} d m \geq \int_{\mathbb{T}}|(c P+d Q) f|^{2} d m .
$$

(2) $|a| \geq|c|,|b| \geq|d|$, and either (a) or (b) holds.

(a) $a d-b c=0$.

(b) $\log |a \bar{b}-c \bar{d}| \in L^{1}$, and there is a $V$ in $(H S)(r)$ such that $(a \bar{b}-c \bar{d}) / V$ is in $H^{1 / 2}$, where $r=|a d-b c| /|a \bar{b}-c \bar{d}|$.

Proof. (1) implies that

$$
\int_{\mathbb{T}}\left|a f_{1}+b f_{2}\right|^{2} d m \geq \int_{\mathbb{T}}\left|c f_{1}+d f_{2}\right|^{2} d m .
$$

Let $W_{1}=|a|^{2}-|c|^{2}, W_{2}=|b|^{2}-|d|^{2}$, and let $\phi=a \bar{b}-c \bar{d}$. Then $W_{1}, W_{2}$ are real functions in $L^{1}$, and $\phi$ is a function in $L^{1}$ such that for all $f_{1} \in \mathcal{P}_{1}$ and $f_{2} \in \mathcal{P}_{2}$,

$$
\int_{\mathbb{T}}\left\{\left|f_{1}\right|^{2} W_{1}+\left|f_{2}\right|^{2} W_{2}+2 \operatorname{Re}\left(f_{1} \overline{f_{2}} \phi\right)\right\} d m \geq 0
$$

This is equivalent to the condition (1) of Theorem 2.4. Since $|\phi|^{2}-W_{1} W_{2}=$ $|a d-b c|^{2}$ and

$$
r^{2}=\frac{|\phi|^{2}-W_{1} W_{2}}{|\phi|^{2}}=\frac{|a \bar{b}-c \bar{d}|^{2}-\left(|a|^{2}-|c|^{2}\right)\left(|b|^{2}-|d|^{2}\right)}{|a \bar{b}-c \bar{d}|^{2}}=\left|\frac{a d-b c}{a \bar{b}-c \bar{d}}\right|^{2}
$$


this theorem follows from Lemma 2.3. This completes the proof.

Remark B. For a function $r$ satisfying $0 \leq r \leq 1$ and $\int_{\mathbb{T}} r d m>0$,

$$
(H S)(r)=\left\{W \in L^{1}: W>0, \int_{\mathbb{T}}|f|^{2} W d m \geq \int_{\mathbb{T}}|r P f|^{2} W d m,(f \in \mathcal{P})\right\} .
$$

Proof. Let $a=b=\sqrt{W}, c=r \sqrt{W}$ and $d=0$. By Theorem 2.4, $W / V \in H^{1 / 2}$. By Neuwirth-Newman's theorem (cf. [14], p.79), $W / V$ is a constant, so $W \in$ $(H S)(r)$. The converse is also true.

Theorem 2.5. Let $W$ be a positive function in $L^{1}$. Let $a, b, c, d$ be in $L^{\infty}$. Then the following conditions (1) and (2) are equivalent.

(1) For all $f$ in $\mathcal{P}$,

$$
\int_{\mathbb{T}}|(a P+b Q) f|^{2} W d m \geq \int_{\mathbb{T}}|(c P+d Q) f|^{2} W d m
$$

(2) $|a| \geq|c|,|b| \geq|d|$, and either (a) or (b) holds.

(a) $a d-b c=0$.

(b) $\log |a \bar{b}-c \bar{d}| W \in L^{1}$, and there is a $V$ in $(H S)(r)$ such that $(a \bar{b}-$ $c \bar{d}) W / V$ is in $H^{1 / 2}$, where $r=|a d-b c| /|a \bar{b}-c \bar{d}|$.

Proof. Suppose (1) holds and (a) of (2) does not hold. Let $a_{1}=a \sqrt{W}, b_{1}=$ $b \sqrt{W}, c_{1}=c \sqrt{W}, d_{1}=d \sqrt{W}$. Then $\int_{\mathbb{T}}\left|a_{1} d_{1}-b_{1} c_{1}\right| d m>0$ and

$$
\int_{\mathbb{T}}\left|\left(a_{1} P+b_{1} Q\right) f\right|^{2} d m \geq \int_{\mathbb{T}}\left|\left(c_{1} P+d_{1} Q\right) f\right|^{2} d m
$$

for all $f$ in $\mathcal{P}$. By Theorem 2.4, this implies that $\log \left|a_{1} \overline{b_{1}}-c_{1} \bar{d}_{1}\right| \in L^{1},\left|a_{1}\right|^{2}-$ $\left|c_{1}\right|^{2} \geq 0,\left|b_{1}\right|^{2}-\left|d_{1}\right|^{2} \geq 0$, and there is a $V$ in $(H S)(r)$ such that $\left(a_{1} \bar{b}_{1}-c_{1} \bar{d}_{1}\right) / V$ is in $H^{1 / 2}$, where $r=\left|a_{1} d_{1}-b_{1} c_{1}\right| /\left|a_{1} \bar{b}_{1}-c_{1} \bar{d}_{1}\right|=|a d-b c| /|a \bar{b}-c \bar{d}|$. Hence (b) of (2) holds, so (1) implies (2). The converse is also true.

Remark C. Let $W$ be a positive function in $L^{1}$. Let $L^{2}(W)$ be the weighted Lebesgue space with the norm

$$
\|f\|_{2, W}=\left\{\int_{\mathbb{T}}|f|^{2} W d m\right\}^{1 / 2} .
$$

When $W=1$, then we write $\|f\|=\|f\|_{W}$. Let $A=a P+b Q$, and let $B=$ $c P+d Q$. Then the condition (1) implies that $B$ is majorized by $A$ on $L^{2}(W)$, i.e., $A^{*} A \geq B^{*} B$ on $L^{2}(W)$, i.e., $\|A f\|_{2, W} \geq\|B f\|_{2, W}$ for all $f$ in $L^{2}(W)$.

\section{Applications of Theorem 2.4}

The equivalence of (1) and (3) of the following corollary is Widom-DevinatzRochberg's theorem (cf. [1], [7], [6], [13], p.250, [15], p.93). Nakazi [7] removed the condition $W \in(H S)$ and established the condition of $\alpha$ satisfying

$$
\int_{\mathbb{T}}|(\alpha P+Q) f|^{2} W d m \geq \varepsilon^{2} \int_{\mathbb{T}}|P f|^{2} W d m,
$$

for all $f \in \mathcal{P}$. 
Corollary 3.1. ([7]) Let $W$ be in $(H S)$ and let $\alpha$ be in $L^{\infty}$. Then the following are equivalent.

(1) $T_{\alpha}$ is bounded below on $H^{2}(W)$.

(2) $\alpha^{-1} \in L^{\infty}$, and there is a $V$ in $(H S)$ such that $\alpha W / V$ is in $H^{1 / 2}$.

(3) $\alpha^{-1} \in L^{\infty}$, and there is an inner function $q$ and a real function $t \in L^{1}$ such that $\alpha /|\alpha|=q e^{i \tilde{t}}$ and $W e^{-t} \in(H S)$.

Proof. By (1), there is a constant $\varepsilon>0$ such that

$$
\int_{\mathbb{T}}|P(\alpha P f)|^{2} W d m=\int_{\mathbb{T}}\left|T_{\alpha}(P f)\right|^{2} W d m \geq \varepsilon^{2} \int_{\mathbb{T}}|P f|^{2} W d m,
$$

for all $f \in \mathcal{P}$. Since $W \in(H S), P$ is bounded on $L^{2}(W)$, so

$$
\begin{gathered}
\|P\|_{W}^{2} \int_{\mathbb{T}}|(\alpha P+Q) f|^{2} W d m \geq \int_{\mathbb{T}}|P(\alpha P+Q) f|^{2} W d m \\
=\int_{\mathbb{T}}|P(\alpha P f)|^{2} W d m \geq \varepsilon^{2} \int_{\mathbb{T}}|P f|^{2} W d m,
\end{gathered}
$$

for all $f \in \mathcal{P}$. Let $a=\|P\|_{W} \alpha \sqrt{W}, b=\|P\|_{W} \sqrt{W}, c=\varepsilon \sqrt{W}$ and $d=0$. Then

$$
\left.\int_{\mathbb{T}}|(a P+b Q) f|^{2} d m \geq \int_{\mathbb{T}} \mid c P+d Q\right)\left.f\right|^{2} d m .
$$

By Theorem 2.4, $|a| \geq|c|$, so $\|P\|_{W}|\alpha| \geq \varepsilon>0$, and $r=|a d-b c| /|a \bar{b}-c \bar{d}|=$ $\varepsilon /\left(\|P\|_{W}|\alpha|\right) \leq 1$. Since $\alpha \in L^{\infty}, r^{-1} \in L^{\infty}$. By Theorem 2.4, there is a $V$ in $(H S)(r)$ such that $\|P\|_{W}^{2} \alpha W / V=(a \bar{b}-c \bar{d}) / V$ is in $H^{1 / 2}$. Since $V \in(H S)(r)$, $V=\gamma e^{u+\tilde{v}}$, where $u$ and $v$ are real functions such that $u \in L^{1}, v \in L^{\infty},|v| \leq$ $\pi / 2$, and $r^{2} e^{u}+e^{-u} \leq 2 \cos v$. Since $r^{-1} \in L^{\infty}, u \in L^{\infty}$ and $\|v\|_{\infty}<\pi / 2$, so $V \in(H S)$. This implies (2). The converse is also true. Suppose (2) holds. Since $\alpha W / V \in H^{1 / 2}$, there is an inner function $q$ and real function $t \in L^{1}$ such that $\alpha W / V=q e^{t+i \tilde{t}}$. Thus $\alpha /|\alpha|=q e^{i \tilde{t}}$ and $W e^{-t}=V /|\alpha| \in(H S)$. This implies (3). The converse is also true. This completes the proof.

The following corollary is the Feldman-Krupnik-Markus theorem ([6], Vol. 2, p.213, Theorem 5.1, and p.215, Lemma 5.3). $\|\alpha P+\beta Q\|_{W}$ and $\|P\|_{W}$ denote the operator norms of each operators on $L^{2}(W)$. In [11], this theorem was generalized to the case when $\alpha$ and $\beta$ are functions in $L^{\infty}$.

Corollary 3.2. ([6]) Let $\alpha$ and $\beta$ be constants. Let

$$
\gamma=\left|\frac{\alpha-\beta}{2}\right|^{2}\left(\|P\|_{W}^{2}-1\right)
$$

then

$$
\|\alpha P+\beta Q\|_{W}=\sqrt{\gamma+\left(\frac{|\alpha|+|\beta|}{2}\right)^{2}}+\sqrt{\gamma+\left(\frac{|\alpha|-|\beta|}{2}\right)^{2}} .
$$


Proof. We assume that $\|\alpha P+\beta Q\|_{W}^{2} \neq \alpha \bar{\beta}$. Let $a=b=\sqrt{W}\|\alpha P+\beta Q\|_{W}$, $c=\alpha \sqrt{W}$ and $d=\beta \sqrt{W}$. Then for all $f$ in $\mathcal{P}$,

$$
\int_{\mathbb{T}}|(a P+b Q) f|^{2} d m \geq \int_{\mathbb{T}}|(c P+d Q) f|^{2} d m .
$$

By Theorem 2.4, $\log \left|\|\alpha P+\beta Q\|_{W}^{2}-\alpha \bar{\beta}\right|=\log |a \bar{b}-c \bar{d}| \in L^{1}$, and there is a $V$ in $(H S)(r)$ such that $\left(\|\alpha P+\beta Q\|_{W}^{2}-|\alpha \bar{\beta}|\right) W / V=(a \bar{b}-c \bar{d}) / V$ is in $H^{1 / 2}$, where

$$
r=\frac{|a d-b c|}{|a \bar{b}-c \bar{d}|}=\frac{|\alpha-\beta|\|\alpha P+\beta Q\|_{W}}{\left|\|\alpha P+\beta Q\|_{W}^{2}-\alpha \bar{\beta}\right|} .
$$

Since $\|\alpha P+\beta Q\|_{W}^{2} \neq \alpha \bar{\beta}, W / V \in H^{1 / 2}$ and $W / V \geq 0$. By the Neuwirth-Newman theorem (cf. [14], p.79), $W / V$ is a constant, so $W \in(H S)(r)$. By Theorem 2.4

$$
\int_{\mathbb{T}}|f|^{2} W d m \geq \int_{\mathbb{T}}|r P f|^{2} W d m .
$$

Hence $r=1 /\|P\|_{W}$, so $\|P\|_{W}$ is described by $\|\alpha P+\beta Q\|_{W}$. By the calculation, $\|\alpha P+\beta Q\|_{W}$ is described by $\alpha, \beta$ and $\|P\|_{W}$. This completes the proof.

An operator $A$ is called hyponormal if its self-commutator $A^{*} A-A A^{*}$ is positive. If $\alpha-\beta$ is a constant, then the following theorem gives the descriptions of symbols of normal (and hyponormal) operators $\alpha P+\beta Q$. Brown and Halmos ([2]) proved that the Toeplitz operator $T_{\alpha}$ is normal if and only if $\alpha$ satisfies the condition (2) of the following corollary for some $c \in \mathbb{C}$. In [12], normal singular integral operator $\alpha P+\beta Q$ is considered without the condition that $\alpha-\beta$ is a constant.

Corollary 3.3. Let $\alpha$ and $\beta$ be non-constant functions in $L^{\infty}$. Suppose $\alpha-\beta$ is a non-zero constant. Then the following are equivalent.

(1) $\alpha P+\beta Q$ is normal.

(2) $\alpha=c f+\bar{f}+b$ for some $f \in z H^{2}$ and $b \in \mathbb{C}$, where $c=(\alpha-\beta) /(\bar{\alpha}-\bar{\beta})$.

(3) $\alpha P+\beta Q$ is hyponormal.

Proof. $(3) \Rightarrow(1)$ : By (3), $\|(\alpha P+\beta Q) f\|^{2} \geq\left\|(\alpha P+\beta Q)^{*} f\right\|^{2}$, for all $f \in L^{2}$. Since $\alpha-\beta \in \mathbb{C}$, it follows that

$$
(\alpha P+\beta Q)^{*}=((\alpha-\beta) P+\beta I)^{*}=(\bar{\alpha}-\bar{\beta}) P+\bar{\beta} I=\bar{\alpha} P+\bar{\beta} Q .
$$

Thus $\|\alpha P f+\beta Q f\|^{2} \geq\|\bar{\alpha} P f+\bar{\beta} Q f\|^{2}$. Hence $2 \operatorname{Re} \int_{\mathbb{T}}(\alpha \bar{\beta}-\bar{\alpha} \beta) P f \overline{Q f} d m \geq 0$, for all $f \in L^{2}$. This implies that $2 \operatorname{Re} \int_{\mathbb{T}}(\alpha \bar{\beta}-\bar{\alpha} \beta) P f \overline{Q f} d m=0$, for all $f \in L^{2}$. Thus $\|(\alpha P+\beta Q) f\|^{2}=\left\|(\alpha P+\beta Q)^{*} f\right\|^{2}$, for all $f \in L^{2}$. Therefore $\alpha P+\beta Q$ is normal.

$(1) \Rightarrow(3)$ : Trivial.

$(3) \Leftrightarrow(2)$ : There exists a complex constant $c$ such that $\beta=\alpha+c$. By Theorem 2.4 and the above proof, if $\alpha \bar{c}-\bar{\alpha} c=0$, then (3) and (2) are equivalent. By Theorem 2.4, if $\alpha \bar{c}-\bar{\alpha} c \neq 0$, then (3) and (2) are equivalent, because $(H S)(1)$ is the set of all positive constants, and the real function $i(\alpha \bar{c}-\bar{\alpha} c)$ belongs to $H^{1 / 2} \cap L^{\infty}=H^{\infty}$, so that it is a real constant. 
Acknowledgement. The author is supported by Research Grant in HokkaiGakuen University.

\section{REFERENCES}

1. A. Böttcher and B. Silbermann, Analysis of Toeplitz Operators, Akademie-Verlag, Berlin, and Springer-Verlag, 1990.

2. A. Brown and P.R. Halmos, Algebraic properties of Toeplitz operators, J. Reine Angew. Math. 213(1963/1964), 89-102.

3. M. Cotlar and C. Sadosky, On the Helson-Szegö theorem and a related class of modified Toeplitz kernels, pp. 383-407, Harmonic analysis in Euclidean spaces, Part 1, Proc. Symp. Pure Math. 35, Amer. Math. Soc., Providence, 1979.

4. R.G. Douglas, On majorization, factorization, and range inclusion of operators on Hilbert space, Proc. Amer. Math. Soc. 17 (1966), 413-415.

5. J. Garnett, Bounded Analytic Functions, Academic Press, New York, 1981.

6. I. Gohberg and N. Krupnik, One-dimensional Linear Singular Integral Equations, Vol. 1-2, Birkhäuser Verlag, Basel, 1992.

7. T. Nakazi, Toeplitz operators and weighted norm inequalities, Acta Sci. Math. (Szeged) $\mathbf{5 8}$ (1993), 443-452.

8. T. Nakazi and T. Yamamoto, Some singular integral operators and Helson-Szegö measures, J. Funct. Anal. 88 (1990), 366-384.

9. T. Nakazi and T. Yamamoto, Weighted norm inequalities for some singular integral operators, J. Funct. Anal. 148 (1997), 279-295.

10. T. Nakazi and T. Yamamoto, Norms of some singular integral operators on weighted $L^{2}$ spaces, J. Operator Theory 50 (2003), 311-330.

11. T. Nakazi and T. Yamamoto, Norms and essential norms of the singular integral operator with Cauchy kernel on weighted Legesgue spaces, Integral Equations Operator Theory 68 (2010), 101-113.

12. T. Nakazi and T. Yamamoto, Normal singular integral operators with Cauchy kernel on $L^{2}$, preprint.

13. N.K. Nikolski, Operators, Functions, and Systems: An Easy Reading, Vol. 1-2, Amer. Math. Soc., Providence, 2002.

14. D. Sarason, Sub-Hardy Hilbert spaces in the unit disk, John Wiley \& Sons, 1994.

15. K. Seip, Interpolation and Sampling in Spaces of Analytic Functions, Amer. Math. Soc., Providence, 2004.

16. T. Yamamoto, Boundedness of some singular integral operators in weighted $L^{2}$ spaces, J. Operator Theory 32 (1994), 243-254.

Department of Mathematics, Hokkai-Gakuen University, Sapporo 062-8605, JAPAN.

E-mail address: yamamoto@elsa.hokkai-s-u.ac.jp 